\title{
Influence of impurities and surface defects on the flux-induced current in mesoscopic $d$-wave superconducting loops
}

\author{
Guo-Qiao Zha, ${ }^{1,2}$ M. V. Milošević, ${ }^{1}$ Shi-Ping Zhou, ${ }^{2}$ and F. M. Peeters ${ }^{1}$ \\ ${ }^{1}$ Departement Fysica, Universiteit Antwerpen, Groenenborgerlaan 171, B-2020 Antwerpen, Belgium \\ ${ }^{2}$ Department of Physics, Shanghai University, Shanghai 200444, China
}

(Received 21 April 2011; revised manuscript received 14 September 2011; published 7 October 2011)

\begin{abstract}
We investigated the magnetic flux dependence of the supercurrent in mesoscopic $d$-wave superconducting loops, containing impurities and surface defects, by numerically solving the Bogoliubov-de Gennes equations self-consistently. In the presence of impurities, bound states arise close to the Fermi energy. In the case of a single impurity, the flux-induced current is found to be suppressed. This can be different when more impurities are introduced in the sample due to the quantum interference effect, which depends sensitively on the relative position between the impurities. We further analyze the effect of small surface defects at the inner or outer edge of the loop, and show that indentation and bulge defects have pronounced and different effects on the supercurrent.
\end{abstract}

DOI: 10.1103/PhysRevB.84.132501

PACS number(s): 74.20.Rp, 73.23.Ra, 74.78.Na

\section{INTRODUCTION}

The $h c / e$ periodicity of the circulating supercurrent in superconducting flux-threaded mesoscopic systems has been numerically investigated recently ${ }^{1-9}$ based on different models. For nodal superconductors, such as $d$-wave superconducting square loops, it is known that the ground-state energy is generically $h c / e$ periodic due to the lifted degeneracy in energy of two distinct supercurrent-carrying states. ${ }^{1,8}$

The effect of impurities on conventional and unconventional superconductors has also attracted considerable interest both experimentally and theoretically for many years as it may be a valuable probe of the mechanism of superconductivity. ${ }^{10}$ In addition to the traditional investigations of the effect of impurities on bulk properties, a recent study has shown that nonmagnetic impurities as well as pairing correlations strongly affect the superconducting properties of an $s$-wave nanoring, and the actual impurity configuration is of vital importance for the magnitude of the persistent current. ${ }^{11}$ However, it has remained unaddressed as to how impurities influence the properties of nanosized $d$-wave superconductors and, in particular, the magnetic flux periodicity of $h c / e$ in nanorings, because scattering of nodal quasiparticles on impurities is drastic. On the other hand, it is known that imperfections and defects of the mesoscopic samples have strong impact on, e.g., vortex entry and exit in mesoscopic disks, ${ }^{12}$ or symmetry-induced vortex states in superconducting squares ${ }^{13}$ and squares with holes. ${ }^{14}$ Although bound to be interesting, phenomena related to impurities and defects in magnetic flux-threaded $d$-wave loops have not been considered to date.

In this Brief Report, we systematically investigate the flux-induced supercurrent in mesoscopic $d$-wave loops by solving the Bogoliubov-de Gennes (BdG) equations in a self-consistent manner. We generalize the method of Ref. 8 for clean systems and consider the presence of impurities and surface defects. Our numerical analysis concerns the supercurrent as a function of the magnetic flux in the presence of a single nonmagnetic impurity when the impurity scattering strength and the impurity site are varied. We also discuss the effect of interference due to scattering on many impurities on the supercurrent, where interference is clearly sensitive on the exact position of the impurities. Finally, we address the influence of defects in the inner or outer edge of the $d$-wave loop on the persistent supercurrent in the sample.

\section{THEORY}

We start with the pairing Hamiltonian by assuming nearestneighbor attraction $V$ for $d$-wave superconducting (DSC) pairing:

$$
\begin{aligned}
H= & -\sum_{\langle\mathbf{i} j\rangle, \sigma} t_{\mathbf{i} j} \exp \left(i \varphi_{\mathbf{i j}}\right) c_{\mathbf{i} \sigma}^{\dagger} c_{\mathbf{j} \sigma}+\sum_{\mathbf{i}, \sigma}\left(V_{0} \delta_{\mathbf{i}, \mathbf{i}_{m}}-\mu\right) c_{\mathbf{i} \sigma}^{\dagger} c_{\mathbf{i} \sigma} \\
& +\sum_{\langle\mathbf{i} j\rangle}\left(\Delta_{\mathbf{i} j} c_{\mathbf{i} \uparrow}^{\dagger} c_{\mathbf{j} \downarrow}^{\dagger}+\Delta_{\mathbf{i} j}^{*} c_{\mathbf{j} \downarrow} c_{\mathbf{i} \uparrow}\right) .
\end{aligned}
$$

Here, $t_{\mathrm{i} j}=t$ are the nearest-neighbor hopping integrals, $c_{\mathrm{i} \sigma}$ $\left(c_{\mathbf{i} \sigma}^{\dagger}\right)$ are destruction (creation) operators for electron of spin $\sigma, n_{\mathbf{i} \sigma}=c_{\mathbf{i} \sigma}^{\dagger} c_{\mathbf{i} \sigma}$ is the number operator, and $\mu$ is the chemical potential determining the averaged electron density. $\mathbf{i}_{m}$ denotes the position of the impurity site, and $V_{0}$ is the single-site potential describing the scattering from nonmagnetic impurities. The Peierl's phase factor is given by $\varphi_{\mathbf{i} j}=$ $2 \pi / \Phi_{0} \int_{r_{\mathrm{i}}}^{r_{\mathrm{j}}} \mathbf{A}(r) \cdot d \mathbf{r}$ with the flux quantum $\Phi_{0}=h c / e$. Using the Bogoliubov transformation $c_{\mathbf{i} \sigma}=\sum_{n}\left[u_{\mathbf{i} \sigma}^{n} \gamma_{n \sigma}-\sigma v_{\mathbf{i} \sigma}^{n *} \gamma_{n \bar{\sigma}}^{\dagger}\right]$, the Hamiltonian in Eq. (1) can be diagonalized by solving the resulting BdG equations self-consistently:

$$
\sum_{\mathbf{j}}^{N}\left(\begin{array}{cc}
\mathcal{H}_{\mathbf{i} j \sigma} & \Delta_{\mathbf{i} j} \\
\Delta_{\mathbf{i} j}^{*} & -\mathcal{H}_{\mathbf{i} j \bar{\sigma}}^{*}
\end{array}\right)\left(\begin{array}{c}
u_{\mathbf{j} \sigma}^{n} \\
v_{\mathbf{j} \bar{\sigma}}^{n}
\end{array}\right)=E_{n}\left(\begin{array}{c}
u_{\mathbf{i} \sigma}^{n} \\
v_{\mathbf{i} \bar{\sigma}}^{n}
\end{array}\right),
$$

where $\mathcal{H}_{\mathbf{i} j \sigma}=-t_{\mathbf{i} j}+\left[V_{0} \delta_{\mathbf{i}, i_{m}}-\mu\right] \delta_{\mathbf{i} j}$. Note that, with respect to bulk superconductors, the translational symmetry is broken for finite mesoscopic systems. With the open boundary conditions, we can get the eigenvalues $\left\{E_{n}\right\}$ with eigenvectors $\left\{\mathrm{u}^{n}, \mathrm{v}^{n}\right\}$. The current density $J_{i j}$ from lattice site $i$ to $j$ is given by

$$
\begin{aligned}
J_{\mathbf{i} j}= & -4 \frac{e t}{\hbar c} \sum_{n} \Im\left(\left\{\mathbf{u}_{\mathbf{j}}^{n} \mathbf{u}_{\mathbf{i}}^{n *} f\left(E_{n}\right)\right.\right. \\
& \left.\left.+\mathbf{v}_{\mathbf{j}}^{n *} \mathbf{v}_{\mathbf{i}}^{n}\left[1-f\left(E_{n}\right)\right]\right\} \exp \left(i \varphi_{\mathbf{i j}}\right)\right),
\end{aligned}
$$




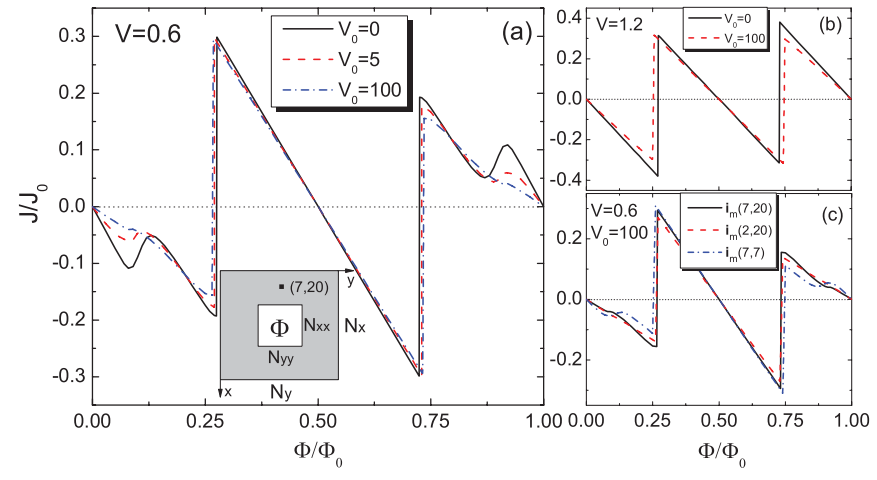

FIG. 1. (Color online) Total supercurrent (in units of $J_{0}=e t / \hbar c$ ) as a function of the magnetic flux $\Phi$ in units of $\Phi_{0}=h c / e$ for different impurity scattering strength $V_{0}$ with $V=0.6$ (a) and $V=1.2$ (b) in a square $N_{x} \times N_{y}=40 \times 40$ loop with a centered $N_{x x} \times N_{y y}=12 \times$ 12 hole. The inset in (a) shows schematically the two-dimensional square loop, which is threaded by a magnetic field $\Phi$ in the hole. The nonmagnetic impurity is placed at the site $\mathbf{i}_{m}(7,20)$. (c) Total supercurrent as a function of magnetic flux for different impurity sites $\mathbf{i}_{m}$ with $V=0.6$ and $V_{0}=100$ in the same square loop.

where $f(E)=\left(e^{E / k_{B} T}+1\right)^{-1}$ is the Fermi-Dirac distribution function. For more details on the numerical and analytical approaches used in this Brief Report, we refer the reader to Ref. 8 .

\section{SINGLE IMPURITY}

First, we consider a symmetric square $d$-wave loop as schematically shown in the inset of Fig. 1(a) with a size of $N_{x} \times N_{y}=40 \times 40$ and a centered hole of size $N_{x x} \times N_{y y}=$ $12 \times 12$, which is threaded by a magnetic flux $\Phi$ in the hole. We introduce a nonmagnetic impurity such as $\mathrm{Zn}$ into our system at site $\mathbf{i}_{m}(7,20)$ and investigate its effect on the flux-induced current. Figure 1(a) plots the total supercurrent as a function of magnetic flux for different impurity scattering strength $V_{0}=0$ (black solid line), 5 (red dashed line), and 100 (dashed-dotted line). The pairing interaction is chosen as $V=0.6$. One can easily see that the circulating supercurrent oscillates as a function of the magnetic flux with an observable period of $\Phi_{0}=h c / e$. For different $V_{0}$, we find that a single impurity always reduces the persistent current. The zigzaglike behavior of the flux-dependent current due to the nodal character of the $d$-wave order parameter is dramatically suppressed with increasing impurity strength. In contrast to conventional $s$-wave superconductors, in $d$-wave systems, a finite nonmagnetic impurity potential acts as a pair breaker that can modify dramatically the density of states close to the Fermi level and induce a virtual bound state. ${ }^{10}$ Therefore, the activation behavior in the supercurrent is replaced by a more linearlike behavior. In Fig. 2, the eigenenergies below the Fermi energy $E_{F}=0$ in the gap region corresponding to the impurity scattering strength $V_{0}=5$ and 100 are shown. Some well-separated discrete states are present due to the nodal character of the order parameter. As a result of the reconstruction of the energy spectrum of the condensate for different winding numbers $q$ of the order parameter and the flux-driven change in the quasiparticle subgap states, in both cases we find a $\Phi_{0}=h c / e$ periodicity. In the presence of impurities, the bound state close to $E_{F}$ arises, which is not

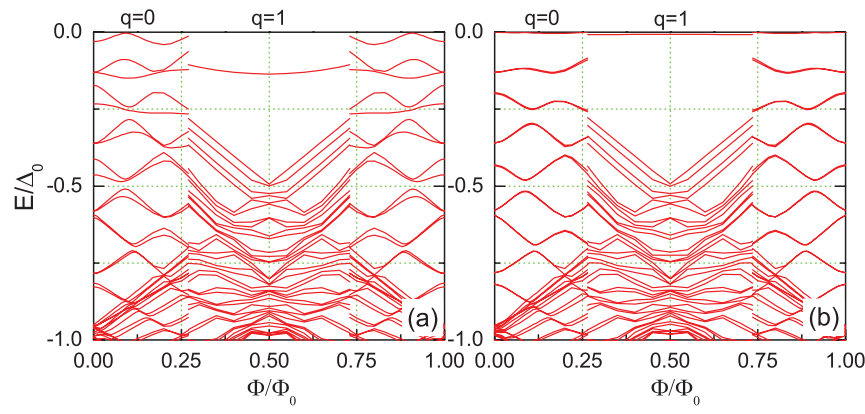

FIG. 2. (Color online) The eigenenergies (in units of the average superconducting order parameter $\Delta_{0}$ at $\Phi=0$ ) in the gap region as a function of the magnetic flux are shown for an impurity with $V_{0}=5$ (a) and $V_{0}=100$ (b) in a square loop. The other parameter values are the same as those in Fig. 1(a). $q$ is the winding number of the order parameter, and there is a clear difference between condensate states with $q=0$ and 1 .

present in the case without impurities (see Fig. 1 in Ref. 8). Moreover, we notice that the highest bound state is pushed toward the Fermi energy as the impurity potential strength is increased.

For a larger $d$-wave pairing interaction $V=1.2$, the corresponding evolution of the supercurrent with magnetic field is plotted in Fig. 1(b). It is shown that the system evolves toward a state where the supercurrent exhibits $\Phi_{0} / 2$ periodicity for an enlarged gap regime (black solid line), and almost shows a $\Phi_{0} / 2$-periodic behavior in the presence of a strong nonmagnetic impurity (dashed line). Note that the current is not exactly $\Phi_{0} / 2$ periodic, which is a mesoscopic effect corresponding to the not exact $\Phi_{0}$ periodicity in $s$-wave loops. ${ }^{15}$ In addition, the flux dependence of the supercurrent for different impurity sites $\mathbf{i}_{m}$ is displayed in Fig. 1(c). We note that, compared to a single impurity placed at the midway point of the arm (black solid line), an impurity near the loop's edge (dashed line) or in the diagonal (dashed-dotted line) can play a stronger or weaker role in the flux dependence of the current, and the zigzag feature vanishes or remains present.

\section{MANY IMPURITIES}

Next, we extend the analysis to the effect of many nonmagnetic impurities. More systematical investigations are given in this section in comparison to the $s$-wave system. ${ }^{11}$ In the presence of many impurities, the wave function of electrons bound to the separate impurities interfere at long distances, leading to a collective behavior that can no longer be described as independent additions of single independent impurities. Figure 3 presents the flux dependence of the persistent supercurrent for several configurations of two impurities (a) as well as many random impurities (b) with $V_{0}=5$. In the $d$-wave case, the overlapping and interference of many impurity states can lead to a splitting of bound-state energies and an accumulation of low-energy impurity-induced energy eigenvalues, i.e., the so-called "impurity band." 16 The formation of the impurity band is strongly influenced by the fact that significant overlaps between two impurity states can take place only if the impurities are "oriented" with respect to one another such that the nodal quasiparticle wave 

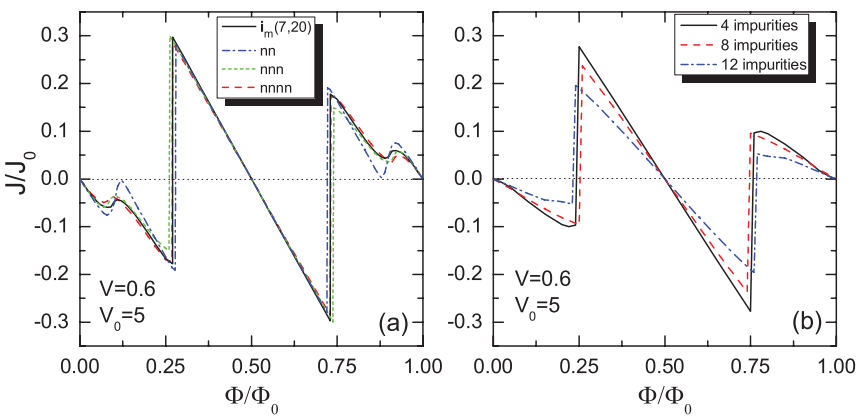

FIG. 3. (Color online) Total supercurrent as a function of the magnetic flux for different situations where (a) two impurities and (b) many random impurities are placed in a square $40 \times 40$ loop with a centered $12 \times 12$ hole. The calculation is performed with $V=0.6$ and $V_{0}=5$. "nn," "nnn," and "nnnn" denote an additional impurity placed at the nearest-neighbor, next-nearest-neighbor, and third-nearest-neighbor site of a single impurity at site $\mathbf{i}_{m}(7,20)$, respectively.

functions overlap along the [110] direction. ${ }^{17}$ In Fig. 3(a), for two impurities located at the nearest neighbor (nn), the persistent current shows more pronounced zigzag oscillations than in the case of a single impurity at site $\mathbf{i}_{m}(7,20)$, due to the cancellation effect of the two nn impurities (see the dashed-dotted line). Figure 4(a) depicts the corresponding energy spectrum. We notice a clear large jump in the energy spectrum close to $\Phi=h c / 4 e$ when $q$ changes from $q=0$ to 1 , which is similar to the spectrum of the clean frame discussed in Ref. 8 (Fig. 1). This however, depends on the relative position of the impurities. For two impurities placed at the next-nearest-neighbor (nnn) and third-nearest-neighbor (nnnn) sites, the oscillations of the persistent current are slightly increased and decreased due to the destructive and constructive interference effect, respectively [see the dotted and dashed lines in Fig. 3(a)].

With the addition of few random impurities, the supercurrent becomes smaller with increasing the impurity concentration, as shown in Fig. 3(b). This figure also shows the disappearance of the zigzag features of the supercurrent as a function of the flux for sufficiently large impurity concentrations. In Fig. 4(b), the corresponding energy spectrum for 12 random impurities is displayed. Note that the empty energy gap in the $q=1$ regime almost disappears due to a population

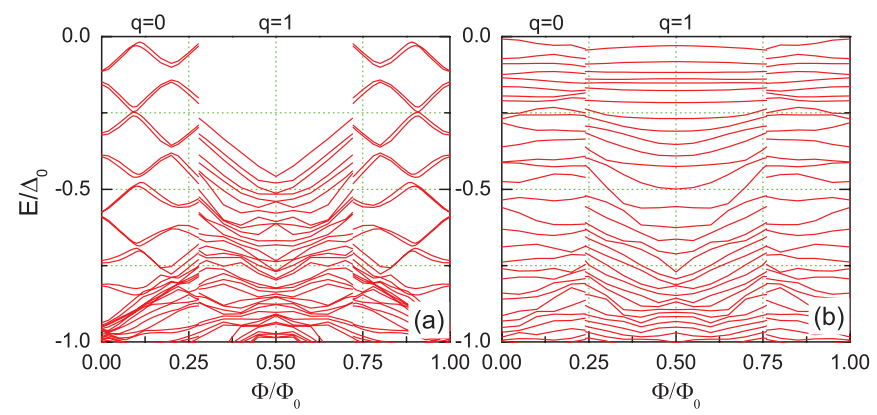

FIG. 4. (Color online) The eigenenergies in the gap region as a function of the magnetic flux are shown for (a) $2 \mathrm{nn}$ impurities, and (b) 12 random impurities, placed in a square loop. The other parameter values are the same as those in Fig. 3.
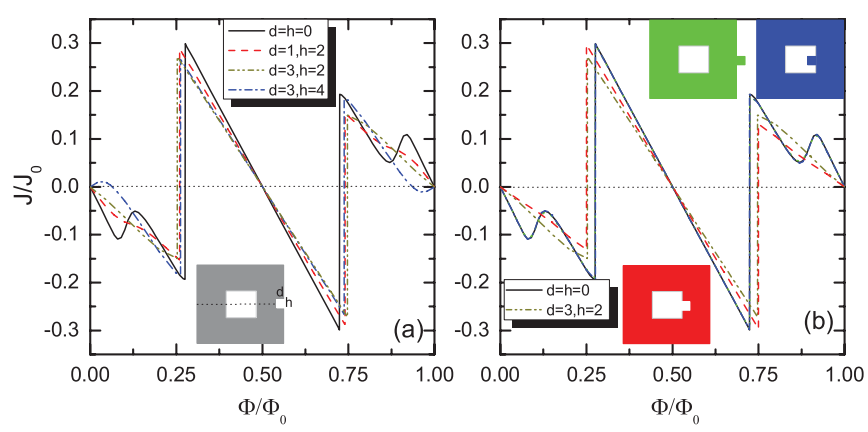

FIG. 5. (Color online) Total supercurrent as a function of the magnetic flux for a square $40 \times 40$ loop with a centered $12 \times 12$ hole and (a) an indentation with different sizes $d$ and $h$ at the middle of the outer edge, (b) an indentation or a bulge with the same $d$ and $h$ at the middle of the inner or outer edge. The insets in (b) show schematically two-dimensional square loops with different kinds of defects, with the same colors as those of the corresponding curves.

of the low-energy excitations, and the $\Phi_{0}$ periodic behavior becomes less pronounced.

\section{SURFACE DEFECTS}

Finally, we examine the influence of surface defects on the flux-induced current in mesoscopic $d$-wave superconducting loops. In this study, we restrict ourselves to defects that are small indentations and bulges at the surface of the sample. It is known that such defects may influence the penetration and expulsion of magnetic flux as was demonstrated experimentally by Geim et al. in Ref. 18. The defect under study is first placed at the center of the edge of the square loop and is taken to be of rectangular shape with size $d \times h$. The results for a square $40 \times 40$ loop with a centered $12 \times 12$ hole are shown in Fig. 5. In Fig. 5(a), the corresponding evolution of supercurrent with magnetic flux for a square loop with indentations of different sizes is shown. The flux-induced supercurrent is clearly sensitive to an indentation. For defects with fixed width $h$ but different length $d$, the activationlike zigzag feature is depressed when the side $d$ is increased. Interestingly, enlarged $h$ can lead to opposite directions of the current around $\Phi=0$ when $d$ is fixed (see the dashed-dotted line), which indicates that the occupied level closest to $E_{F}$ with an orbital moment opposite to the applied magnetic field that starts to dominate.

For other kinds of defects with the same sizes, such as a indentation at the inner boundary as well as a bulge at either the inner or outer boundary, the results are shown in Fig. 5(b). We conclude that the supercurrent is more sensitive to an indentation at the inner edge (see the dashed line) than one at the outer boundary of the sample. However, bulge and indentation defects do not have a similar effect on the flux-induced current. By contrast, bulge defects at inner and outer edges both have weak effect (see the dashed-dotted and dotted lines, respectively), and the corresponding evolution of supercurrent with flux is very similar to that of the case without defects (solid line).

Aside from the defect located exactly in the center of the sample edge, we also investigated defects that are displaced 


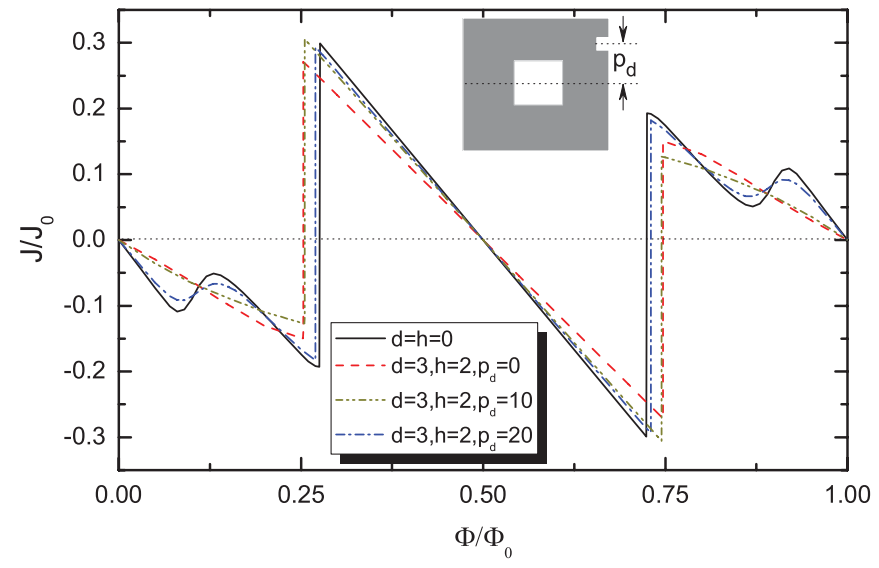

FIG. 6. (Color online) Total supercurrent as a function of the magnetic flux for a square $40 \times 40$ loop with a centered $12 \times 12$ hole and an indentation defect with different distances $p_{d}$ from the edge center.

to an off-center location. Precisely, we studied the influence of the position of an edge indentation defect with $d=3$ and $h=2$ on the supercurrent, depending on the defect's distance $p_{d}$ from the edge center. The result is shown in Fig. 6 with the dashed-dot-dotted line for $p_{d}=10$ and the dashed-dotted line for $p_{d}=20$ (i.e., a defect at the corner). As the defect is shifted further from the center of the sample edge, we observed an increase of current in the $q=1$ state, while for the $q=0$ state the zigzag oscillatory behavior appears again.

\section{CONCLUSIONS}

In summary, we have investigated the magnetic flux dependence of supercurrent in mesoscopic $d$-wave superconducting loops with impurities and surface defects by numerically solving the BdG equations self-consistently. In the presence of impurities, we have demonstrated the existence of bound states close to the Fermi energy. The flux-induced current is generally suppressed for the single impurity case. However, the opposite can be found when many impurities are introduced, depending on the relative position of impurities and their quantum interference. Furthermore, we find that small surface defects strongly affect the current. The flux-induced supercurrent is generally more sensitive to an indentation than to a bulge. Notice that in real $d$-wave superconductors, the surface roughness is on atomistic length scale but nevertheless modifies the properties of the quasiparticles since the coherence length of the samples is comparable to the lattice constant of the material. The BdG equations in a two-dimensional lattice have been used to study the effect of surface roughness in Ref. 19, and the results of the numerical calculations with rough surfaces are consistent with experiment. We expect that our theoretical results will be useful for future experiments, where rough surfaces, which can be modeled by randomly placing strong impurities near the surface, are inevitable. We also would like to emphasize that the chosen grid size in our self-consistent calculation is optimal to obtain reliable results in minimal time. We checked other grid sizes, and no significant variations are found for the effect of impurities for different sample sizes.

\section{ACKNOWLEDGMENTS}

This work was supported by the Flemish Science Foundation (FWO-Vl), by Belgian Science Policy (IAP), by National Science Foundation of China (Grant Nos. 10904089 and 60971053), and by research funds under Grant Nos. 20093108120005, S30105, 09JC1406000, and 10zz63.
${ }^{1}$ F. Loder, A. P. Kampf, T. Kopp, J. Mannhart, C. W. Schneider, and Y. S. Barash, Nat. Phys. 4, 112 (2008).

${ }^{2}$ T.-C. Wei and P. M. Goldbart, Phys. Rev. B 77, 224512 (2008).

${ }^{3}$ V. Vakaryuk, Phys. Rev. Lett. 101, 167002 (2008).

${ }^{4}$ Y. S. Barash, Phys. Rev. Lett. 100, 177003 (2008).

${ }^{5}$ V. Juričić, I. F. Herbut, and Z. Tešanović, Phys. Rev. Lett. 100, 187006 (2008).

${ }^{6}$ F. Loder, A. P. Kampf, and T. Kopp, Phys. Rev. B 78, 174526 (2008).

${ }^{7}$ F. Loder, A. P. Kampf, T. Kopp, and J. Mannhart, New J. Phys. 11, 075005 (2009).

${ }^{8}$ G.-Q. Zha, M. V. Milošević, S.-P. Zhou, and F. M. Peeters, Phys. Rev. B 80, 144501 (2009).

${ }^{9}$ J.-X. Zhu and H. T. Quan, Phys. Rev. B 81, 054521 (2010).

${ }^{10}$ For recent reviews, see A. V. Balatsky, I. Vekhter, and J.-X. Zhu, Rev. Mod. Phys. 78, 373 (2006); H. Alloul, J. Bobroff, M. Gabay, and P. J. Hirschfeld, ibid. 81, 45 (2009).

${ }^{11}$ K. Czajka, M. M. Maska, M. Mierzejewski, and Z. Sledz, Phys. Rev. B 72, 035320 (2005).
${ }^{12}$ B. J. Baelus, K. Kadowaki, and F. M. Peeters, Phys. Rev. B 71, 024514 (2005)

${ }^{13}$ A. S. Melnikov, I. M. Nefedov, D. A. Ryzhov, I. A. Shereshevskii, V. M. Vinokur, and P. P. Vysheslavtsev, Phys. Rev. B 65, 140503 (2002).

${ }^{14}$ R. Geurts, M. V. Milošević, and F. M. Peeters, Phys. Rev. Lett. 97, 137002 (2006); Phys. Rev. B 75, 184511 (2007); 79, 174508 (2009).

${ }^{15}$ B. J. Baelus, F. M. Peeters, and V. A. Schweigert, Phys. Rev. B 61, 9734 (2000).

${ }^{16}$ U. Micheluchi, F. Venturini, and A. Kampf, J. Phys. Chem. Solids 63, 2283 (2002).

${ }^{17}$ J. W. Harter, B. M. Andersen, J. Bobroff, M. Gabay, and P. J. Hirschfeld, Phys. Rev. B 75, 054520 (2007).

${ }^{18}$ A. K. Geim, S. V. Dubonos, I. V. Grigorieva, K. S. Novoselov, F. M. Peeters, and V. A. Schweigert, Nature (London) 407, 55 (2000).

${ }^{19}$ Y. Tanuma, Y. Tanaka, M. Yamashiro, and S. Kashiwaya, Phys. Rev. B 57, 7997 (1998). 